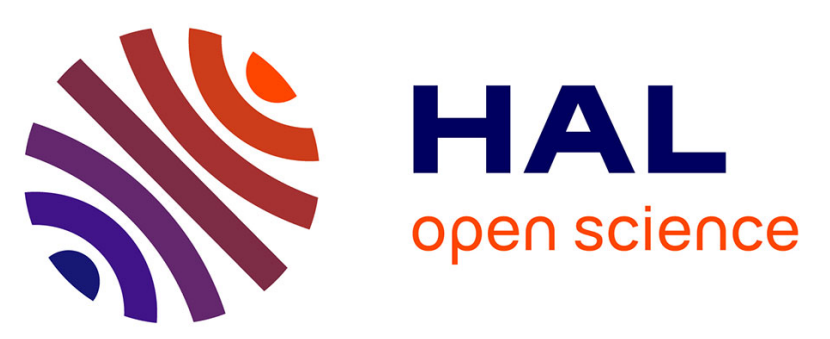

\title{
Static Anti-windup Scheme for a Class of Homogeneous Dwell-time Hybrid Controllers
}

Francesco Fichera, Christophe Prieur, Sophie Tarbouriech, Luca Zaccarian

\section{To cite this version:}

Francesco Fichera, Christophe Prieur, Sophie Tarbouriech, Luca Zaccarian. Static Anti-windup Scheme for a Class of Homogeneous Dwell-time Hybrid Controllers. ECC 2013 - 12th biannual European Control Conference, Jul 2013, Zurich, Switzerland. 6p. hal-00830544

\section{HAL Id: hal-00830544 https://hal.science/hal-00830544}

Submitted on 5 Jun 2013

HAL is a multi-disciplinary open access archive for the deposit and dissemination of scientific research documents, whether they are published or not. The documents may come from teaching and research institutions in France or abroad, or from public or private research centers.
L'archive ouverte pluridisciplinaire HAL, est destinée au dépôt et à la diffusion de documents scientifiques de niveau recherche, publiés ou non, émanant des établissements d'enseignement et de recherche français ou étrangers, des laboratoires publics ou privés. 


\title{
Static Anti-windup Scheme for a Class of Homogeneous Dwell-time Hybrid Controllers
}

\author{
Francesco Fichera, Christophe Prieur, Sophie Tarbouriech and Luca Zaccarian
}

\begin{abstract}
In this paper, some tools for the analysis of a class of hybrid control systems for a continuous-time plant with input magnitude saturation are presented. We show that certain continuous-time LMI-based techniques can be extended to this setting when a dwell-time property is satisfied by the hybrid loop. Moreover, for the case in which the hybrid controller has the same order as the plant, the synthesis of a static direct linear anti-windup (DLAW) compensator is proposed. The need of including the flow and jump sets in the analysis and synthesis conditions leads to LMIs for global results, that is, when the plant is exponentially stable and BMIs otherwise.
\end{abstract}

Index Terms-Hybrid controller, saturation, anti-windup, $\mathcal{L}_{2}$ gain

\section{INTRODUCTION}

Hybrid control theory has attracted several work in order to provide more flexible stabilizing and performing controllers. Such controllers suitably combining continuous and discrete dynamics have been capable to robustly stabilize nonlinear systems, which are not stabilizable by smooth feedback [11], [17]. In [14] promising performance analysis for some specific hybrid systems have been presented with respect to rise-time, overshoot, settling-time. In [16], it is shown that the desirable closed-loop behavior may be induced by resetting the controller according to an optimal reset law. Also in [18], optimal techniques for overshoot reduction and maximization of the decay rate have been presented. Furthermore, by adding a dwell-time structure in the hybrid controller, further flexibility can be used to establish global exponential stability of the origin, [4], [24]. Recently in [2], a convex $\mathcal{H}_{\infty}$ synthesis of a plant-order hybrid controller with guaranteed convergence rate was proposed.

Another important feature is actuator saturation, which is present in all control systems. The magnitude of the signal that an actuator can deliver is usually limited by physical or safety constraints. While these limits restrict the performance achievable by the systems, if these limits are not treated carefully, pernicious behaviors may occur. Roughly speaking, there are two approaches which one could adopt to avoid saturation problems in systems which are known to have actuator limits (see, for example, [21] and references therein). In the current paper, the selected approach is the anti-windup technique, whose general principle is the introduction of

Work supported by HYCON2 Network of Excellence "Highly-Complex and Networked Control Systems", grant agreement 257462.

F. Fichera, S. Tarbouriech and L. Zaccarian are with CNRS, LAAS, 7 avenue du colonel Roche, F-31400 Toulouse, France and Univ. de Toulouse, LAAS, F-31400 Toulouse, France. ffichera@laas.fr, tarbourelaas.fr, zaccarianelaas.fr

L. Zaccarian is also with University of Trento, Italy.

C. Prieur is with Department of Automatic Control, Gipsa-lab, 961 rue de la Houille Blanche, BP 46, 38402 Grenoble Cedex, France, christophe.prieuragipsa-lab. fr an extra feedback loop in a pre-designed control system to mitigate the effects caused by saturation [6], [20], [23], [26].

The current paper proposes preliminary results for a class of hybrid systems with dwell-time logic subject to input magnitude saturation. Such class includes (but not only) hybrid control systems of interest as the ones in [2]-[4] as well as the ones employing a FORE controller as in [14], [15], [25]. Therefore this paper allows the estimate of the region of attraction and the $\mathcal{L}_{2}$-gain estimation once that saturation is taken into account. Regarding the synthesis, the strategy chosen consists in designing a static anti-windup compensator to recover the performance of the pre-designed unconstrained hybrid control system. The synthesis results rely on the strong hypothesis that the controller is plant order. To date, the only optimal syntheses of a plant-order hybrid controller are proposed in [2], [19], therefore the result in here for the synthesis can straightforwardly be applied to those cases. Nevertheless we stress that as long as the plant and the unconstrained hybrid controller have the same order, the anti-windup synthesis in this paper can be applied. The conditions allowing to prove the global exponential stability of the complete closed loop are under an LMI form for exponentially stable plants. On the other hand, for local (regional) exponential stability, the conditions are BMIs. Within the literature, we mention [22], where anti-windup strategies were proposed for SISO systems with a FORE controller. Although the results here cover a wider class of hybrid systems, we stress that the strategies adopted to describe the hybrid loops are different in the two papers, yielding to different conditions whenever the results in here are applied to a SISO plant controlled by a FORE controller. In this sight, this paper completes [22], with alternative conditions. Notice that both this paper and [22] present in some cases BMIs, due to the complexity of the problem.

The paper is structured as follows. In Section II, the control architecture under consideration and the assumptions are stated. Section III presents the main results regarding both stability and performance analysis of the closed loop and the procedure to design static anti-windup gains. In Section IV, a simulation is performed illustrating the interest of the results. A conclusion and some open questions are finally presented in Section V.

Notation. Given a vector $x, x^{T}$ denotes the transpose of $x$. $\mathbb{R}$ denotes the set of real numbers, $\mathbb{R}_{>0}$ denotes the set of non-negative real numbers. For a matrix $M, \operatorname{He}(M)=M+$ $M^{T}$. For a positive integer $n, I_{n}$ (respectively, $0_{n}$ ) denotes the identity matrix (respectively, the null matrix) in $\mathbb{R}^{n \times n}$. The subscripts may be omitted when there is no ambiguity. For any $s \in \mathbb{R}$, the function $\mathrm{dz}: \mathbb{R} \rightarrow \mathbb{R}$ is defined by 
$\mathrm{dz}(s)=0$ if $|s| \leq 1$ and $\mathrm{dz}(s)=\operatorname{sgn}(s)(|s|-1)$ if $|s| \geq 1$. Given the vector $\bar{u}=\left[\bar{u}_{1}, \ldots, \bar{u}_{n_{u}}\right]^{T} \in \mathbb{R}^{m}$ with $\bar{u}_{i}>0$, $i=1, \ldots, n_{u}$, the saturation function is defined as

$$
\operatorname{sat}_{\bar{u}_{i}}\left(u_{i}\right):=\left\{\begin{aligned}
\bar{u}_{i}, & u_{i} \geq \bar{u}_{i}, \\
u_{i}, & u_{i} \in\left[-\bar{u}_{i}, \bar{u}_{i}\right], \\
-\bar{u}_{i}, & u_{i} \leq-\bar{u}_{i}
\end{aligned}\right.
$$

and $\operatorname{sat}(u)=\left[\operatorname{sat}_{\bar{u}_{1}}\left(u_{1}\right) \ldots \operatorname{sat}_{\bar{u}_{m}}\left(u_{m}\right)\right]^{T}$, while $q=$ $u-\operatorname{sat}(u)$.

\section{PROBLEM STATEMENT}

We consider the linear time-invariant plant $\mathcal{P}$

$$
\begin{aligned}
\dot{x}_{p} & =\bar{A}_{p} x_{p}+\bar{B}_{p} \sigma+\bar{B}_{w} w \\
z & =\bar{C}_{z} x_{p}+\bar{D}_{z} \sigma+\bar{D}_{z w} w \\
y & =\bar{C}_{p} x_{p}+\bar{D}_{p} \sigma+\bar{D}_{w} w
\end{aligned}
$$

with $x_{p} \in \mathbb{R}^{n_{p}}$ the state of the plant, $\sigma \in \mathbb{R}^{n_{u}}$ the control input subject to saturation nonlinearity, $w \in \mathbb{R}^{n_{w}}$ an exogenous signal, $z \in \mathbb{R}^{n_{z}}$ the performance output and $y \in \mathbb{R}^{n_{y}}$ the measured output.

With reference to Figure 1, we assume that for the continuous-time plant $\mathcal{P}$ in (1) a hybrid controller $\mathcal{K}$ has been designed corresponding to the following dynamics with $x=\left(x_{p}, x_{c}\right)$

$$
\begin{aligned}
& \left\{\begin{aligned}
\dot{x}_{c} & =\bar{A}_{c} x_{c}+\bar{B}_{c} y+\bar{B}_{c w} w+v_{1} \\
\dot{\tau} & =1-\mathrm{dz}\left(\frac{\tau}{\rho}\right)
\end{aligned} \quad(x, \tau) \in \mathcal{C}\right. \\
& \left\{\begin{array}{l}
x_{c}^{+}=G_{p} x_{p}+G_{c} x_{c} \\
\tau^{+}=0
\end{array} \quad(x, \tau) \in \mathcal{D}\right. \\
& u=\bar{C}_{c} x_{c}+\bar{D}_{c} y+\bar{D}_{c w} w+v_{2}
\end{aligned}
$$

with $x_{c} \in \mathbb{R}^{n_{c}}, u \in \mathbb{R}^{n_{u}}, v_{i}=0, i=1,2$ (those inputs will be used later for anti-windup design). The sets $\mathcal{C}$ and $\mathcal{D}$ in (2a) are selected based on the following symmetric cones, defined by the matrix $M=M^{T} \in \mathbb{R}^{\left(n_{p}+n_{c}\right) \times\left(n_{p}+n_{c}\right)}$

$$
\begin{aligned}
& \mathcal{F}=\left\{x \in \mathbb{R}^{n_{p}+n_{c}}: x^{T} M x \leq 0\right\}, \\
& \mathcal{J}=\left\{x \in \mathbb{R}^{n_{p}+n_{c}}: x^{T} M x \geq 0\right\} .
\end{aligned}
$$

In particular, we choose

$$
\begin{aligned}
\mathcal{C} & =(\mathcal{F} \times[0,2 \rho]) \cup\left(\mathbb{R}^{n_{p}+n_{c}} \times[0, \rho]\right), \\
\mathcal{D} & =\mathcal{J} \times[\rho, 2 \rho],
\end{aligned}
$$

which can be equivalently written as (see [4], [14], [15], [25] for results using the notation in (2e))

$$
\begin{aligned}
\mathcal{C} & =\{(x, \tau): x \in \mathcal{F} \text { or } \tau \in[0, \rho]\}, \\
\mathcal{D} & =\{(x, \tau): x \in \mathcal{J} \text { and } \tau \in[\rho, 2 \rho]\} .
\end{aligned}
$$

We make the following standing assumptions.

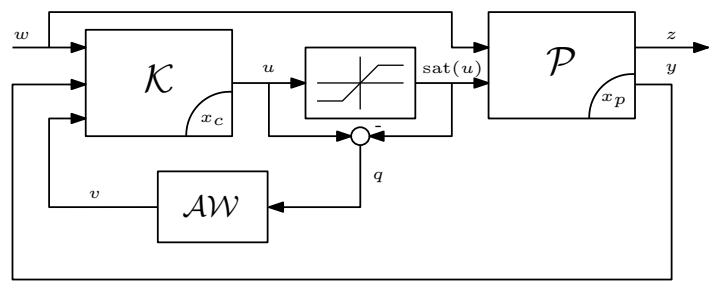

Fig. 1. The classical anti-windup compensation scheme.
Assumption 1: For system (2), the reset map $G$ and the flow and jump sets $\mathcal{F}$ and $\mathcal{J}$ in (2b) and (2c), are such that $\forall x \in \mathcal{J} \Longrightarrow G x \in \mathcal{F}$.

Assumption 2: The hybrid unconstrained closed loop between controller (2) with $\left(v_{1}, v_{2}\right)=0$ and plant (1) with $\sigma=u$ is well posed (namely, $\left(I-\bar{D}_{c} \bar{D}_{p}\right)$ is non-singular) and its origin is GES.

Remark 1: Note that using [24, Proposition 1] due to homogeneity, the GES property of Assumption 2 is guaranteed under the weaker assumption that the origin is LAS.

Note that $\mathcal{C} \cup \mathcal{D}=\mathbb{R}^{\left(n_{p}+n_{c}\right) \times\left(n_{p}+n_{c}\right)} \times[0,2 \rho]$ and the system data satisfy the basic closed-loop conditions of [8] so that solutions exist for all initial conditions of $\left(x_{p}, x_{c}\right)$ and for all initial values in $[0,2 \rho]$ for the timer $\tau$.

As noted in [1, Proposition 1.1], the dynamics of the timer $\tau$ in (2a) and the jump set in (2d) ensure that each pair of consecutive jumps is spaced by at least $\rho$ amount of flow in the ordinary time direction. More precisely, and using the hybrid systems framework of [7], [8], each solution $\xi=$ $(x, \tau)$ to (1)-(2) has a hybrid domain $E=\operatorname{dom}(\xi)$ such that any two elements $(t, j),(s, k)$ of $E$ with $t>s$ satisfy $t-s \geq \rho(j-k)$.

In this paper we will propose anti-windup techniques to recover possible stability and performance loss when saturation occurs at the plant input, namely $\sigma=\operatorname{sat}(u)$. More specifically, $\operatorname{sat}(\cdot)$ is the symmetric vector saturation function with limits $\bar{u}=\left[\bar{u}_{1}, \ldots, \bar{u}_{n_{u}}\right]^{T 1}$. We will use inputs $v_{1}, v_{2}$ to design regional and global direct linear anti-windup (DLAW) following similar tools to those currently available for linear control systems (see [6], [20], [26]). To this aim, the above commented dwell time is a fundamental property that motivates the use of ordinary-time $\mathcal{L}_{2}$ norm (just as in [2], [5], [15]) defined as ${ }^{2}$

$$
\|\xi\|_{2 t}=\left(\sum_{j \in \operatorname{dom}_{j}(\xi)} \int_{t_{j}}^{t_{j+1}}|\xi(t, j)|^{2} d t\right)^{\frac{1}{2}},
$$

(with $t_{j+1}$ possibly being $\infty$ if $(j+1) \notin \operatorname{dom}_{j}(\xi)$ ) where $\operatorname{dom}_{j}(\xi)=\{j \in \mathbb{N}:(t, j) \in \operatorname{dom}(\xi)$ for some $t \geq 0\}$. The definition in (3) essentially corresponds to the continuoustime $\mathcal{L}_{2}$ norm of the continuous-time signal $\xi_{t}(t)$ obtained by projecting on the ordinary time the dwell-time hybrid signal $\xi(t, j)$. Note that when the hybrid signal $\xi$ only flows, that is $\operatorname{dom}(\xi)=[0,+\infty) \times\{0\}$, then (3) corresponds to the standard continuous-time $\mathcal{L}_{2}$ norm. Note also that (3) is not a norm because, for example, a solution $\xi$ starting at a nonzero position and jumping to zero at $(t, j+1)=(0,1)$ would satisfy $\|\xi\|_{2 t}=0$. Nevertheless we call it norm through the paper due to the intuition that it generalizes the continuoustime norm.

Controller (2) for plant (1) with $\sigma=u$ (namely, considering the unconstrained case) may arise from many hybrid control scheme designs. In particular, it includes all

\footnotetext{
${ }^{1}$ Only symmetric saturation functions with respect to the amplitude of the signal $u$ will be considered. Nevertheless asymmetric saturations can be treated with the methods here introducing some conservativeness by selecting $\bar{u}_{i}$ as the minimum absolute value of the negative and positive saturation levels.

${ }^{2}$ For a summary of the notation used here, the reader is referred to [7], or the summary in [2].
} 
the controllers in our previous works [2]-[4], as well as the FORE controller architecture, see [14], [15], [25]. The analysis and synthesis tools that will be presented in the sequel can certainly be applied to all these schemes and this justifies the interest for the results here presented.

\section{STATIC ANTI-WINDUP SYNTHESIS}

\section{A. Overview}

In this section we will use DLAW techniques to recover stability and performance by way of a static anti-windup gain which will use inputs $v_{1}$ and $v_{2}$ in (2), namely we will only affect the flow dynamics of the control scheme. To this aim, it will be useful to characterize in a compact way the closed loop (1)-(2) with no anti-windup, namely with $\left(v_{1}, v_{2}\right)=0$. The resulting dynamics is given by

$$
\left\{\begin{array}{l}
\dot{x}=A x+B_{q} q+B_{w} w \\
\dot{\tau}=1-\mathrm{dz}\left(\frac{\tau}{\rho}\right) \\
x^{+}=G x \\
\tau^{+}=0 \\
u=C_{u} x+D_{u q} q+D_{u w} w \\
z=C_{z} x+D_{z q} q+D_{z w} w \\
q=u-\operatorname{sat}(u)
\end{array} \quad(x, \tau) \in \mathcal{D}\right.
$$

with $x=\left[x_{p}^{T} x_{c}^{T}\right] \in \mathbb{R}^{n}$, sets $\mathcal{C}$ and $\mathcal{D}$ defined as in (2e) and where, defining $\chi:=\left(I-\bar{D}_{c} \bar{D}_{p}\right)^{-1}$ (which is well defined from Assumption 2),

$$
\begin{aligned}
& {\left[\begin{array}{c}
A \\
\hline \frac{G}{C_{u}} \\
\hline C_{z}
\end{array}\right]=\left[\begin{array}{cc}
\bar{A}_{p}+\bar{B}_{p} \chi \bar{D}_{c} \bar{C}_{p} & \bar{B}_{p} \chi \bar{C}_{c} \\
\bar{B}_{c}\left(\bar{C}_{p}+\bar{D}_{p} \chi \bar{D}_{c} \bar{C}_{p}\right) & \bar{A}_{c}+\bar{B}_{c} \bar{D}_{p} \chi \bar{C}_{c} \\
\hline I & 0 \\
G_{p} & G_{c} \\
\hline \chi \bar{D}_{c} \bar{C}_{p} & \chi \bar{C}_{c} \\
\hline \bar{C}_{z}+\bar{D}_{z} \chi \bar{D}_{c} \bar{C}_{p} & \bar{D}_{z} \chi \bar{C}_{c}
\end{array}\right]} \\
& {\left[\begin{array}{c|c}
B_{q} & B_{w} \\
\hline D_{u q} & D_{u w} \\
\hline D_{z q} & D_{z w}
\end{array}\right]=\left[\begin{array}{c|c}
-\bar{B}_{p} \chi & \bar{B}_{p} \chi \Xi_{d}+\bar{B}_{w} \\
-\bar{B}_{c} \bar{D}_{p} \chi & \bar{B}_{c} \bar{D}_{p} \chi \Xi_{d}+\bar{B}_{c} \bar{D}_{w}+\bar{B}_{c w} \\
\hline I-\chi & \chi \Xi_{d} \\
\hline-\bar{D}_{z} \chi & \bar{D}_{z} \chi \Xi_{d}+\bar{D}_{z w}
\end{array}\right],}
\end{aligned}
$$

with $\Xi_{d}:=\bar{D}_{c} \bar{D}_{w}+\bar{D}_{c w}$.

Based on (3), we will denote by $t$ - $\mathcal{L}_{2}$ gain from $w$ to $z$ the worst case ratio between $\|z\|_{2 t}$ and $\|w\|_{2 t}$ over all $w$ such that $w \in t-\mathcal{L}_{2}$ (that is $\|w\|_{2 t}<\infty$ ) whenever (4) starts from zero initial condition (namely, $x(0,0)=0$ ). To suitably characterize anti-windup performance, the $t-\mathcal{L}_{2}$ gain of system (4) from $w$ to $z$ will be considered as a performance index. In addition to the $t-\mathcal{L}_{2}$ gain, we will also characterize the reachable set and domain of attraction of the closed loop, as specified in the next section. We will also use the exponential stability property for hybrid systems, see [24].

\section{B. Analysis}

The following problems will be addressed for system (4) in this section:
1. Estimate of the domain of attraction $(w=0)$ by using a forward invariant ellipsoid $\mathcal{E}\left(s^{-2} P\right):=\left\{x: x^{T} P x \leq\right.$ $\left.s^{2}\right\}$.

2. Estimate of the reachable set from a given bound on the norm of $w$ (i.e., $\|w\|_{2 t}<s$ ) and zero initial condition.

3. Estimate of the nonlinear $t-\mathcal{L}_{2}$ gain from $w$ to $z$ from the same bound on the norm of $w$ and zero initial condition.

The following result generalizes [12, Lemma 1], to the hybrid case considered here.

Proposition 1: Consider system (4) under Assumption $1^{3}$.

1. If there exist $P=P^{T}>0, H \in \mathbb{R}^{n_{u} \times n}$, a diagonal matrix $W>0$, a positive real scalar $s$ and non-negative scalars $\tau_{F}, \tau_{R} \in \mathbb{R}_{\geq 0}$ such that

$$
\begin{aligned}
& \operatorname{He}\left(\left[\begin{array}{cc}
P A-\tau_{F} M & P B_{q} \\
W H+W C_{u} & W D_{u q}-W
\end{array}\right]\right)<0, \\
& G^{T} P G-P+\tau_{R} M \leq 0, \\
& {\left[\begin{array}{cc}
P & H_{i}^{T} \\
H_{i} & \frac{\bar{u}_{i}^{2}}{s^{2}}
\end{array}\right]>0, \quad \forall i=1, \ldots, n_{u},}
\end{aligned}
$$

with $H_{i}$ denoting the $i$-th row of $H$, then there exists $\bar{\rho}>0$, such that for any $\rho \in(0, \bar{\rho})$, the set $\{0\} \times[0,2 \rho]$ of system (4) is exponentially stable with stability region containing the set $\mathcal{E}\left(s^{-2} P\right) \times[0,2 \rho]$.

2. If there exist $P=P^{T}>0, H \in \mathbb{R}^{n_{u} \times n}$, a diagonal matrix $W>0$, a positive real scalar $s$ and non-negative scalars $\tau_{F}, \tau_{R} \in \mathbb{R}_{\geq 0}$ satisfying (7), (8) and

$\mathrm{He}\left(\left[\begin{array}{ccc}P A-\tau_{F} M & P B_{q} & P B_{w} \\ W H+W C_{u} & W D_{u q}-W & W D_{u w} \\ 0 & 0 & -\frac{1}{2} I\end{array}\right]\right)<0$,

then there exists $\bar{\rho}>0$, such that for any $\rho \in(0, \bar{\rho})$, $\xi(0,0)=(x(0,0), \tau(0,0)) \in\{0\} \times[0,2 \rho]$ and $\|w\|_{2 t} \leq s$, all solutions to (4) satisfy $\xi(t, j) \in \mathcal{E}\left(s^{-2} P\right) \times[0,2 \rho]$ for all $(t, j) \in \operatorname{dom} \xi$.

3. If there exist $P=P^{T}>0, H \in \mathbb{R}^{n_{u} \times n}$, a diagonal matrix $W>0$, positive real scalars $\bar{\gamma}, s$ and non-negative scalars $\tau_{F}, \tau_{R} \in \mathbb{R}_{\geq 0}$ satisfying (7), (8) and

$\operatorname{He}\left(\left[\begin{array}{cccc}P A-\tau_{F} M & P B_{q} & P B_{w} & 0 \\ W H+W C_{u} & W D_{u q}-W & W D_{u w} & 0 \\ 0 & 0 & -\frac{1}{2} I & 0 \\ C_{z} & D_{z q} & D_{z w} & -\frac{\bar{\gamma}^{2}}{2} I\end{array}\right]\right)<0$,

then for any $\gamma$ satisfying

$$
\gamma \geq \bar{\gamma}, \quad \gamma>\sqrt{3\left|D_{z w}\right|^{2}+24 \frac{\left|D_{z q}\right|^{2}\left|W D_{u w}\right|^{2}}{\lambda_{\min }(\Sigma)}},
$$

with $\Sigma:=-\operatorname{He}\left(W\left(D_{u q}-I\right)\right)$, there exists $\bar{\rho}>0$, such that for any $\rho \in(0, \bar{\rho}), \xi(0,0)=(x(0,0), \tau(0,0)) \in$ $\{0\} \times[0,2 \rho]$ and $\|w\|_{2 t} \leq s$, all solutions to (4) satisfy $\|z\|_{2 t} \leq \gamma\|w\|_{2 t}$, namely the $t-\mathcal{L}_{2}$ gain from $w$ to $z$ is less than or equal to $\gamma$.

\footnotetext{
${ }^{3}$ Note that Proposition 1 holds for any system (4) with flow and jump sets (2e) satisfying Assumption 1, disregarding the definitions in (5). Indeed Proposition 1 refers to a wider class of hybrid systems than the one we are addressing for the purpose here.
} 
Item 3 of Proposition 1 implies the previous two items and their implications (the same for item 2 with respect to item 1). It is possible to solve conditions in Proposition 1 by maximizing $s$ to enlarge the domain of attraction or, for a given $s$, minimizing $\gamma$.

Conditions in (11) are needed in item 3 to guarantee that $\bar{\rho}$ exists strictly positive. Although it may look complicated at first sight, it is enough to compare a posteriori $\bar{\gamma}$ with the square root in the second hand term of the latter in (11). If $\bar{\gamma}$ is larger, then we can select $\gamma=\bar{\gamma}$, otherwise we select $\gamma$ larger but arbitrarily close to the value of the square root in the second condition in (11).

Constraints (6), (9) and (10) are not LMIs due to the product between $W$ and $H$. Nevertheless, this nonlinearity only appears if one seeks for regional solutions as clarified in the corollary below. The BMIs for the regional analysis arise from the fact that since (4) is hybrid, it is convenient (and sometimes necessary) to consider the flow and jump sets by taking the matrix $M$ (namely, (2b) and (2c)) into account through two non-negative variables $\tau_{F}$ and $\tau_{R}$. This requires a different proof technique than the one for the linear case (see for instance [9]) which would return stronger nonlinearities also for the global case.

Corollary 1: Consider system (4) under Assumption 1. If there exist $P=P^{T}>0$, a diagonal $W>0$ and non-negative scalars $\tau_{F}, \tau_{R} \geq 0$ satisfying (6) and (7) with $H=0$, then

1. the set $\{0\} \times[0,2 \rho]$ of (4) is GES;

2. there exists a large enough $\bar{\gamma}$ satisfying (10) with $H=0$, and the $t-\mathcal{L}_{2}$ gain of (4) from $w$ to $z$ is less than or equal to $\gamma$, selected according to (11), for all $w \in t-\mathcal{L}_{2}$.

\section{Static DLAW synthesis}

In this section we design a linear static DLAW controller with full authority or external architecture (see [26, Section 2.3.6]) for the interconnection (1)-(2) with $\sigma=\operatorname{sat}(u)$. The proposed technique only applies to the case where the controller has the same order as the plant. Indeed, only in this case it is possible to apply non trivial transformations and provide tractable numerical techniques for the design (LMIs for global properties and BMIs for regional ones). Nevertheless in [2], [19] optimal syntheses for unconstrained plant-order hybrid controllers have been proposed, therefore the results in these section can directly be applied to those techniques.

According to Figure 1, the anti-windup compensator $\mathcal{A W}$ generates correction signals $v$ corresponding to

$$
\left[\begin{array}{l}
v_{1} \\
v_{2}
\end{array}\right]=\left[\begin{array}{ll}
\bar{B}_{c v} & 0 \\
\bar{D}_{c v} & I
\end{array}\right] D_{a w} q,
$$

where the two matrices $\bar{B}_{c v}$ and $\bar{D}_{c v}$ characterize the specific $\mathcal{A} \mathcal{W}$ architecture. In particular, for sake of generality, we will carry out computations that cover the following two cases (see also [26, Section 2.3.6])

- full-authority anti-windup with $\bar{B}_{c v}=I_{n_{c}}$ and $\bar{D}_{c v}=0$ (namely, $v_{1} \in \mathbb{R}^{n_{c}}$ and $v_{2} \in \mathbb{R}^{n_{u}}$ );

- external anti-windup with $\bar{B}_{c v}=\bar{B}_{c}$ and $\bar{D}_{c v}=\bar{D}_{c}$ (namely, $v_{1} \in \mathbb{R}^{n_{y}}$ and $v_{2} \in \mathbb{R}^{n_{u}}$ ).
Note that in the sequel the $\mathcal{A W}$ architecture is supposed to be known.

The static DLAW (12) for the hybrid saturated closed loop results in a very simple anti-windup action only consisting of an additional gain and without requiring any extra dynamics. However, there are stringent conditions that need to be satisfied for this synthesis to be feasible, which are formalized next.

Assumption 3: Controller (2) is plant order, namely $n_{c}=$ $n_{p}$. Moreover, the closed-loop (1)-(2) with $\sigma=u$ is quadratically stable, namely there exists $\bar{P}=\bar{P}^{T}>0, \tau_{F}, \tau_{R} \geq 0$ such that

$$
\begin{array}{r}
\operatorname{He}\left(\bar{P} A-\tau_{F} M\right)<0, \\
G^{T} \bar{P} G-\bar{P}+\tau_{R} M \leq 0 .
\end{array}
$$

The requirements in Assumption 3 clearly restrict the set of control systems where we can apply the techniques of this section. Note however that the control designs of [2], [19] satisfy the requirement $n_{c}=n_{p}$. Moreover, the necessary conditions (13) are stronger than the GES requirement in Assumption 2 (or its relaxation to LAS in Remark 1). Indeed, it is well known that for homogeneous hybrid systems LAS of the origin does not imply the existence of a quadratic Lyapunov function (see, e.g., the counter example in [25, Section 4.1]). Note also that conditions (13) are not yet sufficient for the existence of an anti-windup gain, indeed extra matching conditions similar to those reported in [9], [13] need to hold for feasibility of static anti-windup.

We can write the anti-windup closed-loop dynamics (1), (2), (12) and $\sigma=\operatorname{sat}(u)$ as follows (recall that $x=$ $\left.\left[x_{p}^{T} x_{c}^{T}\right]^{T}\right)$

$$
\begin{aligned}
& \left\{\begin{array}{l}
\dot{x}=A x+\left(B_{c l, q}+B_{c l, v} D_{a w}\right) q+B_{w} w \\
\dot{\tau}=1-\mathrm{dz}\left(\frac{\tau}{\rho}\right)
\end{array}\right. \\
& \left\{\begin{aligned}
x^{+} & =G x \\
\tau^{+} & =0 \\
u & =C_{u} x+\left(D_{c l, u q}+D_{c l, u v} D_{a w}\right) q+D_{u w} w \\
z & =C_{z} x+\left(D_{c l, z q}+D_{c l, z v} D_{a w}\right) q+D_{z w} w
\end{aligned}(x, \tau) \in \mathcal{D}\right.
\end{aligned}
$$

where $A, G, C_{u}, C_{z}, B_{w}, D_{u w}, D_{z w}, \chi, \mathcal{C}$ and $\mathcal{D}$ are the same as those in (2), (5) and

$\left[\begin{array}{c|c}B_{c l, q} & B_{c l, v} \\ \hline D_{c l, u q} & D_{c l, u v} \\ \hline D_{c l, z q} & D_{c l, z v}\end{array}\right]=\left[\begin{array}{c|cc}-\bar{B}_{p} \chi & \bar{B}_{p} \chi \bar{D}_{c v} & \bar{B}_{p} \chi \\ -\bar{B}_{c} \bar{D}_{p} \chi & \bar{B}_{c v}+\bar{B}_{c} \bar{D}_{p} \chi \bar{D}_{c v} & \bar{B}_{c} \bar{D}_{p} \chi \\ \hline I-\chi & \chi \bar{D}_{c v} & \chi \\ \hline-\bar{D}_{z} \chi & \bar{D}_{z} \chi \bar{D}_{c v} & \bar{D}_{z} \chi\end{array}\right]$.

Notice that $\chi$ is non-singular due to Assumption 2 and that system (15) has the same structure as in (4).

Based on the above definitions we can state the following feasibility theorem, which uses equations (14) at the top of the page. If the conditions of Theorem 1 hold, than an antiwindup compensator can be designed using the procedure given at the end of the section.

Theorem 1: Consider (1)-(2) with $\sigma=\operatorname{sat}(u)$ under Assumptions 1, 2 and 3 and denote $M:=\left[\begin{array}{cc}M_{1} & M_{2} \\ M_{2}^{T} & M_{3}\end{array}\right]$. Select $s>0$ and two sets $\mathcal{R}_{p}=\mathcal{E}\left(R_{p}\right)=\left\{x_{p}: x_{p}^{T} R_{p} x_{p} \leq 1\right\}$ and 


$$
\begin{gathered}
\operatorname{He}\left(\left[\begin{array}{ccc}
\left(P_{1}-P_{2}\right)\left(\bar{A}_{p}-\bar{B}_{p}\left(H_{1}-H_{2}\right)\right)-\frac{\tau_{F}}{2}\left(M_{1}-M_{2}^{T}-M_{2}+M_{3}\right) & \left(P_{1}-P_{2}\right) \bar{B}_{w} & 0 \\
0 & -\frac{1}{2} I & 0 \\
\bar{D}_{z w} & -\frac{\bar{\gamma}^{2}}{2} I
\end{array}\right]\right)<0 \\
\bar{C}_{z}-\bar{D}_{z}\left(H_{1}-H_{2}\right) \\
\operatorname{He}\left(\left[\begin{array}{ccc}
P A-\frac{\tau_{F}}{2} M & P B_{w} & 0 \\
0 & -\frac{1}{2} I & 0 \\
C_{z} & D_{z w} & -\frac{\bar{\gamma}^{2}}{2} I
\end{array}\right]\right)<0
\end{gathered}
$$

$\mathcal{S}_{p}=\mathcal{E}\left(S_{p}\right)=\left\{x_{p}: x_{p}^{T} S_{p} x_{p} \leq 1\right\}$, where $R_{p}=R_{p}^{T}>0$ and $S_{p}=S_{p}^{T}>0$.

If there exist $P=P^{T}:=\left[\begin{array}{ll}P_{1} & P_{2} \\ P_{2} & P_{2}\end{array}\right]>0, H:=\left[\begin{array}{ll}H_{1} & H_{2}\end{array}\right]$, $\bar{\gamma}^{2}>0$ and $\tau_{F}, \tau_{R} \geq 0$ such that (7), (8), (14) and $s^{2} R_{p} \leq$ $P_{1} \leq s^{2} S_{p}$ are satisfied, then there exist $\bar{\rho}>0$ and a static anti-windup compensator such that, for any $\rho \in(0, \bar{\rho})$ :

a. the set $\{0\} \times[0,2 \rho]$ is exponentially stable with basin of attraction $\mathcal{E}\left(s^{-2} P\right) \times[0,2 \rho]$,

b. the basin of attraction in the $x_{p}$-direction includes $\mathcal{S}_{p}$,

c. the reachable set from $\|w\|_{2 t} \leq s$, in the $x_{p}$-direction is included in $\mathcal{R}_{p}$,

d. the $t-\mathcal{L}_{2}$ gain from $w$ to $z$ less than or equal to $\gamma$, selected according to (11), for all $w$ such that $\|w\|_{2 t} \leq s$.

Condition $s^{2} R_{p} \leq P_{1}$ (respectively, $s^{2} S_{p} \geq P_{1}$ ) can be ignored if set $\mathcal{R}_{p}$ (respectively, $\mathcal{S}_{p}$ ) is not given. Moreover, Theorem 1 can be solved minimizing $\bar{\gamma}^{2}$, returning an optimal static anti-windup compensator with optimized $t-\mathcal{L}_{2}$ gain, or minimizing $s^{-2}$ to enlarge the basin of attraction.

Remark 2: Note that (7), (8) and (14b), are LMIs, whereas (14a) is a BMI whenever $H \neq 0$ (that is, regional synthesis). As already said for the analysis, such difficulty with respect to the linear case comes from the fact that the flow and jump sets have to be taken into account.

The following corollary parallels Corollary 1 in illustrating that the BMI conditions of Theorem 1 become LMIs if one focuses on global properties.

Corollary 2: Consider (1)-(2) with $\sigma=\operatorname{sat}(u)$ under Assumptions 1, 2 and 3 and denote $M:=\left[\begin{array}{cc}M_{1} & M_{2} \\ M_{2}^{T} & M_{3}\end{array}\right]$.

If $\bar{A}_{p}$ is Hurwitz and there exist $P=P^{T}:=\left[\begin{array}{ll}P_{1} & P_{2} \\ P_{2} & P_{2}\end{array}\right]>0$, $\bar{\gamma}^{2}>0$ and $\tau_{F}, \tau_{R} \geq 0$ such that (7), (14) are satisfied with $H=\left[H_{1} H_{2}\right]=0$, then there exist $\bar{\rho}>0$ and a static antiwindup compensator such that for any $\rho \in(0, \bar{\rho})$ :

1. the set $\{0\} \times[0,2 \rho]$ is GES;

2. the $t-\mathcal{L}_{2}$ gain from $w$ to $z$ is less than or equal to $\gamma$, selected according to (11), for all $w \in t-\mathcal{L}_{2}$.

Corollary 2 is relevant because with $H=0$, (14a) becomes an LMI and the synthesis of a static anti-windup compensator can be achieved with convex tools.

As customary with LMI-based designs arising from the two-step approach exploiting the elimination lemma, once the feasibility conditions of Theorem 1 or Corollary 2 have been solved, an anti-windup compensator can be designed according to the following procedure.

\section{Procedure for static ant-windup synthesis}

Step 1: Find a solution $P, H, \tau_{F}, \tau_{R}, \bar{\gamma}, \gamma$ to the conditions in Theorem 1 (respectively, Corollary 2).

Step 2: Solve

$$
\begin{aligned}
& \operatorname{He}\left(\left[\begin{array}{cccc}
P A-\frac{\tau_{F}}{2} M & P B_{c l, q} U & P B_{w} & 0 \\
H+C_{u} & D_{c l, u q} U-U & D_{u w} & 0 \\
0 & 0 & -\frac{1}{2} I & 0 \\
C_{z} & D_{c l, z q} U & D_{z w} & -\frac{\bar{\gamma}^{2}}{2} I
\end{array}\right]\right) \\
& +\operatorname{He}\left(\left[\begin{array}{c}
P B_{c l, v} \\
D_{c l, u v} \\
0 \\
D_{c l, z v}
\end{array}\right] \Lambda\left[\begin{array}{llll}
0 & I_{n_{u}} & 0 & 0
\end{array}\right]\right)<0,
\end{aligned}
$$

in the unknown $U$ and $\Lambda$, where $U$ is diagonal and positive definite.

Step 3: Select $D_{a w}=\Lambda U^{-1}$.

Remark 3: Practical experience on a few examples indicates that condition (7) may be numerically difficult to satisfy. Indeed, it seems that the non-strict inequality is only solved by the special case where the left hand side of (7) has zero eigenvalues, thus leading to a "thin" feasibility set and inevitable troubles encountered by the LMI solver. A possible workaround to this issue may be to strengthen the flow condition and allow increase in the jump condition, which is probably possible due to the presence of dwell time (see [7, Proposition 3.29]). Then condition (7) would become strict and easier to solve. We regard this extension as future work.

\section{SIMULATIONS}

The synthesis of a DLAW compensator for a hybrid system is presented. In particular, we consider the linear MIMO plant, used also in [10], [26],

$$
\left[\begin{array}{c|c}
\bar{A}_{p} & \bar{B}_{p} \\
\hline \bar{C}_{p} & \bar{D}_{p}
\end{array}\right]=\left[\begin{array}{cc|cc}
-0.01 & 0 & 1 & 0 \\
0 & -0.01 & 0 & 1 \\
\hline-0.4 & 0.5 & 0 & 0 \\
0.3 & -0.4 & 0 & 0
\end{array}\right] .
$$

Note that $\bar{A}_{p}$ is Hurwitz. For our purpose here, we design a hybrid controller with the optimal control technique presented in [2]. To do this, we complete the definition of the plant (with respect to (1)) by selecting $\bar{B}_{w}=\bar{B}_{p}, \bar{D}_{z w}=I$, $\bar{C}_{z}=\bar{C}_{p}, \bar{D}_{z}=I$ and $\bar{D}_{w}=0$.

The obtained optimal hybrid controller is

$$
\begin{aligned}
& {\left[\begin{array}{c|c}
\bar{A}_{c} & \bar{B}_{c} \\
\hline \bar{C}_{c} & \bar{D}_{c}
\end{array}\right]=\left[\begin{array}{cc|cc}
-21.9488 & 1.3106 & -807.10 & -1014.55 \\
2.6550 & -23.4515 & -590.54 & -783.46 \\
\hline 7.6418 & -1.2505 & 267.15 & 332.069 \\
-1.2505 & 8.0351 & 191.03 & 257.877
\end{array}\right],} \\
& {\left[G_{p} \mid G_{c}\right]=\left[\begin{array}{ll|ll}
0.86965 & 0.13213 & 0 & 0 \\
0.13517 & 0.80854 & 0 & 0
\end{array}\right] \text {, }}
\end{aligned}
$$



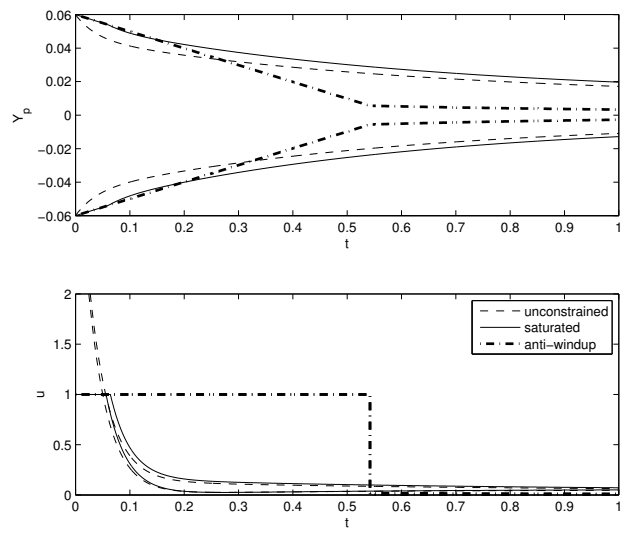

Fig. 2. Input and output responses of various hybrid closed loops.

$$
M=\left[\begin{array}{cccc}
-19.2737 & 21.1029 & 10.4660 & -11.4944 \\
21.1029 & -27.9232 & -11.6764 & 15.2506 \\
10.4660 & -11.6764 & 2.06 \cdot 10^{-8} & 0 \\
-11.4944 & 15.2506 & 0 & 2.06 \cdot 10^{-8}
\end{array}\right]
$$

with $\bar{B}_{c w}=0, \bar{D}_{c w}=0$ and $\rho=5 \cdot 10^{-3}$. The optimal $t-\mathcal{L}_{2}$ gain from $w$ to $z$ is less than or equal to $\gamma=1$. Figure 2 shows the behavior of the unconstrained interconnection (that is, $\sigma=u$ ) of $\mathcal{P}$ and $\mathcal{C}$ with $\left(v_{1}, v_{2}\right)=0$.

Consider now the presence of an actuator with saturation level for each input at \pm 1 . Figure 2 shows that the responses for $\sigma=\operatorname{sat}(u)$ and $\left(v_{1}, v_{2}\right)=0$ present a slower trend than the unconstrained case.

Finally, we satisfy all the conditions of Corollary 2 and through Procedure 1, we obtain the static DLAW compensator in full-authority configuration, with

$$
D_{a w}=\left[\begin{array}{cccc}
0.6650 & 0.3386 & 0.7754 & -0.1010 \\
0.3329 & 0.4472 & -0.0967 & 0.8444
\end{array}\right]^{T},
$$

which guarantees a global $t-\mathcal{L}_{2}$ gain from $w$ to $z$ less than or equal to $\gamma=80.26$.

Notice that the trend in presence of the DLAW compensator is better than the previous two cases. All the simulations start from the initial condition $x_{p}(0,0)=-[0.6,0.6]^{T}$, $x_{c}(0,0)=0$ and $\tau(0,0)=\frac{3}{2} \rho$.

\section{CONCLUSIONS}

A tool for the analysis of a class of hybrid systems with input saturation has been presented. Moreover, a static DLAW compensator design has been proposed to recover (locally or globally) the exponential closed-loop stability in the presence of plant input magnitude saturation whenever the controller is plant order. The static DLAW synthesis is convex for exponentially stable plants, whereas it may be not numerically tractable otherwise.

\section{REFERENCES}

[1] C. Cai, A.R. Teel, and R. Goebel. Smooth Lyapunov Functions for Hybrid Systems, Part II: (Pre)Asymptotically Stable Compact Sets. IEEE Transactions on Automatic Control, 53(3):734-748, 2008.

[2] F. Fichera, C. Prieur, S. Tarbouriech, and L. Zaccarian. A Convex Hybrid $\mathcal{H}_{\infty}$ Synthesis with Guaranteed Convergence Rate. In Proceedings of the 51st Conference on Decision and Control, pages 42174222, Maui (HI), USA, 2012.
[3] F. Fichera, C. Prieur, S. Tarbouriech, and L. Zaccarian. On Hybrid State-feedback Loops Based on a Dwell-time Logic. In 4th IFAC Conference on Analysis and Design of Hybrid Systems, pages 388393, Eindoven, The Netherlands, 2012.

[4] F. Fichera, C. Prieur, S. Tarbouriech, and L. Zaccarian. Using Luenberger Observers and Dwell-time Logic for Feedback Hybrid Loops in Continuous-time Control Systems. International Journal of Robust and Nonlinear Control, 2012 to appear.

[5] F. Forni, D. Nešić, and L. Zaccarian. Reset Passivation of Nonlinear Controllers via Suitable Time-regular Reset Map. Automatica, 47(9):2099-2106, 2011.

[6] S. Galeani, S. Tarbouriech, M. Turner, and L. Zaccarian. A Tutorial on Modern Anti-windup Design. European Journal of Control, (34):418-440, 2009.

[7] R. Goebel, R.G. Sanfelice, and A.R. Teel. Hybrid Dynamical Systems: Modeling, Stability and Robustness. Princeton University Press, 2012

[8] R. Goebel and A.R. Teel. Direct Design of Robustly Asymptotically Stabilizing Hybrid Feedback. ESAIM: Control Optim. Calc. Var. 15(1):205-213, 2009

[9] G. Grimm, J. Hatfield, A.T. Teel, M.C. Turner, and L. Zaccarian. Anti-windup for Stable Linear Systems with Input Saturation: An LMI-Based Synthesis. IEEE Transactions on Automatic Control, 48(9):1509-1525, 2003.

[10] G. Grimm, I. Postlethwaite, A.R. Teel, M.C. Turner, and L. Zaccarian. Regional, Semiglobal, Global Nonlinear Anti-windup via Switching Design. In Proceedings of the European Control Conference, pages 3794-3799, Port, Portugal, 2001.

[11] J.P. Hespanha and A.S. Morse. Stabilization of Nonholonomic Integrators via Logic-based Switching. Automatica, 35(3):385-393, 1999.

[12] T. Hu, A.R. Teel, and L. Zaccarian. Stability and Performance for Saturated Systems via Quadratic and Nonquadratic Lyapunov Functions. IEEE Transactions on Automatic Control, 51(11):1770 1786, November 2006.

[13] T. Hu, A.R. Teel, and L. Zaccarian. Anti-windup Synthesis for Linear Control Systems with Input Saturation: Achieving Regional, Nonlinear Performance. Automatica, 44:512-519, October 2008.

[14] D. Nešić, A.R. Teel, and L. Zaccarian. Stability and Performance of SISO Control Systems with First Order Reset Elements. IEEE Transactions on Automatic Control, 56(11):2567-2582, 2011.

[15] D. Nešić, L. Zaccarian, and A.R. Teel. Stability Properties of Reset Systems. Automatica, 44(8):2019-2026, 2008.

[16] F.R. Poursafei, J.P. Hespanha, and G. Stewart. Quadratic Optimization for Controller Initialization in Multivariable Switching Systems. In Proceedings of the 2010 American Control Conference, pages 25112516, Baltimore, Maryland, USA, June 2010.

[17] C. Prieur. Asymptotic Controllability and Robust Asymptotic Stabilizability. SIAM Journal on Control and Optimization, 43(5):1888-1912, 2005.

[18] C. Prieur, S. Tarbouriech, and L. Zaccarian. Improving the Performance of Linear Systems by adding a Hybrid Loop. In 18th IFAC World Congress, pages 6301-6306, Milano, Italy, September 2011.

[19] A. Satoh. State feedback synthesis of linear reset control with $\mathcal{L}_{2}$ performance bound via lmi approach. In IFAC 18th World Congress, pages 5860-5865, Milan, Italy, 2011.

[20] S. Tarbouriech, G. Garcia, J.M. Gomes da Silva Jr., and I. Queinnec. Stability and Stabilization of Linear Systems with Saturating Actuators. Springer, 2011.

[21] S. Tarbouriech, G. Garcia, and A.H. Glattfelder (Eds.). "Advanced Strategies in Control Systems with Input and Output Constraints.", volume 346 of LNCIS. Springer Verlag, 2007.

[22] S. Tarbouriech, T. Loquen, and C. Prieur. Anti-windup Strategy for Reset Control Systems. International Journal of Robust and Nonlinear Control, 21(10):1159-1177, 2011.

[23] S. Tarbouriech and M.C. Turner. "anti-windup design: an overview of some recent advances and open problems.". IET Control Theory and Application, 3(1):1-19, 2009.

[24] A.R. Teel, F. Forni, and L. Zaccarian. Lyapunov-based Sufficient Conditions for Exponential Stability in Hybrid Systems. IEEE Transactions on Automatic Control, 2012, to appear.

[25] L. Zaccarian, D. Nešić, and A.R. Teel. Analytical and Numerical Lyapunov Functions for SISO Linear Control Systems with Firstorder Reset Elements. International Journal of Robust and Nonlinear Control, 21:1134-1158, 2011.

[26] L. Zaccarian and A.R. Teel. Modern Anti-windup Synthesis. Princeton Press, 2011. 